\title{
Introduction to special issue on verification and evaluation of computer systems
}

\author{
Kamel Barkaoui ${ }^{1} \cdot$ Hanifa Boucheneb ${ }^{2}$
}

Published online: 20 April 2018

(c) Springer-Verlag London Ltd., part of Springer Nature 2018

This special issue of Innovations in Systems and Software Engineering: A NASA Journal is devoted to the selected contributions from the 11th International Conference on Verification and Evaluation of Computer and Communication Systems (VECoS 2017).

The aim of the VECoS conference is to bring together researchers and practitioners in the areas of verification, control, performance and dependability evaluation in order to discuss state-of-the-art and challenges in modern computer and communication systems in which functional and extra-functional properties are strongly interrelated. Thus, the main motivation for VECoS is to encourage the cross-fertilization between various formal verification and evaluation approaches, methods and techniques, and especially those developed for concurrent and distributed hardware/software systems.

VECoS 2017 was organized by Concordia University in Montreal, Quebec, Canada, with the support of Polytechnique Montreal, New Jersey Institute of Technology, Le Cnam and Formal Methods Europe, during August 24-25, 2017.

The Program Committee of VECoS 2017 includes researchers from 20 countries. Among 35 full submissions received from 15 countries, 13 papers were selected for presentation. The conference program also includes 3 invited talks. The invited speakers for VECoS 2017 are: Mourad Debbabi from Concordia University, Montreal, Quebec, Canada; Michel Dagenais from Polytechnique Montreal, Quebec, Canada; and Zhiwu Li from Xidian University,

Kamel Barkaoui

Kamel.barkaoui@cnam.fr

Hanifa Boucheneb

hanifa.boucheneb@polymtl.ca

1 Cedric, Department of Computer Science, Le Cnam, Paris, France

2 Veriform, Department of Computer Engineering, Polytechnique Montreal, Montreal, Canada
China. VECoS 2017 proceedings are published in LNCS volume 10466 by Springer.

The best papers were invited for submission after extension to this special issue of Innovations in Systems and Software Engineering: A NASA Journal, Springer. After a thorough review process, the following 4 papers are accepted for inclusion in this special issue.

The first article titled 'Formal Probabilistic Analysis of a Surgical Robot Control Algorithm with Different Virtual Fixtures' provides a formal analysis of virtual fixture control algorithm, implemented in a neurosurgical robot. A DiscreteTime Markov Chain (DTMC)-based model of the given algorithm and its environment is proposed, and its verification using the PRISM model checker allowed to confirm the safe conditions for the movement of robot for three different virtual fixtures.

The second article titled 'Maximal Software Execution Time: A Regression-based Approach' presents a linear regression method to estimate the maximum execution time (MET) of real-time software through measurements and instrumentation. The authors further use stepwise regression and principal component analysis for automatic model identification. Based on this model, a probabilistic upper bound on the MET is derived. The approach is demonstrated on a JPEG decoder running on a realistic SPARC-V8 processor.

The third paper titled 'TT-BIP: Using Correct-by-Design BIP Approach for Modeling Real-Time System with TimeTriggered Paradigm' proposes the first step of the methodology that links between the model-based design framework Behavior Interaction Priority (BIP) and Time-Triggered (TT) execution platforms. This first step consists in a semanticspreserving transformation of a generic BIP model into a restricted one which is complying with the TT communication primitives. The paper provides formal correctness proofs of the proposed approach as well as its validation on a use case study.

The fourth article titled 'FC-AMAT: Factor-Based CAMAT Analysis in Memory System Measurement' concerns the performance evaluation of the memory systems. After 
exploration of numerical relations between factors' C-AMAT and overall C-AMAT, a method for determining leading factor of a memory system is proposed and thereafter a new C-AMAT model (Concurrent Average Memory Access) that allows an accurate assessment of the effects of a given factor on the average performance of the memory.

The above articles report important recent results in the field of formal verification, validation and evaluation approaches of concurrent systems, and we believe that they will be of great interest for many students and researchers working in these research domains.

As guest editors, we would like to warmly thank the Editor-in-Chief, Prof. Michael G. Hinchey, for giving us the opportunity to host this special issue and for his valuable support. We also express our gratitude to the authors for their contributions and the expert reviewers for their thoughtful, rigorous and helpful work. 